\title{
Impfen ist nicht nur Kindersache!
}

\section{Warum Impfungen auch für Erwachsene wichtig sind}

\author{
Ursula Kunze · Ernest Groman
}

Eingegangen: 18. April 2017 / Angenommen: 18. August 2017 / Online publiziert: 13. September 2017 (C) Der/die Autor(en) 2017. Dieser Artikel ist eine Open-Access-Publikation.

Zusammenfassung Impfungen gehören weltweit zu den zehn erfolgreichsten Präventionsmaßnahmen. Während Impfprogramme für Kinder in Europa installiert sind, ist das Konzept der Erwachsenenimpfung nicht etabliert. Dabei sind Impfungen für Erwachsene überaus sinnvoll: die zunehmende Lebenserwartung läßt uns älter und damit empfänglicher für Infektionskrankheiten werden, Gesundheitsprobleme und Multimorbiditäten werden zunehmen. Die Krankheitslast infolge impfpräventabler Erkrankungen ist in Europa nach wie vor hoch. Infolge der Immunoseneszenz sind Ältere schlechter gegen $\mathrm{Pa}$ thogene geschützt, Antikörpertiter nach Impfungen niedriger und die Dauer der Schutzwirkung kürzer. In Europa gibt es eklatanten Mangel an Daten zu Durchimpfungsraten bei Erwachsenen und zudem keinen internationalen Konsensus bezüglich Impfempfehlungen oder Richtlinien. Nur sechs Länder verfügen über ein ausführliches Dokument, das empfohlene Impfungen für Erwachsene beschreibt, darunter Österreich. Das Bewusstsein um die Wichtigkeit von Impfungen über die gesamte Lebensspanne ist vor allem in Europa noch nicht in dem Maße vorhanden, wie es nötig wäre und muss gefördert werden.

Schlüsselwörter Impfung · Impfen · Erwachsene · Alter · Lebenslanges Impfen

U. Kunze $(\bowtie) \cdot$ E. Groman

Institute of Social Medicine, Center for

Public Health, Medical University of Vienna,

Kinderspitalgasse 15, 1090 Vienna, Österreich

ursula.kunze@meduniwien.ac.at
Immunizing is not only a children's matter! Why vaccinations are also important for adults

Summary Vaccinations belong to the ten most effective public health achievements worldwide. While immunization programms for children are installed in Europe, vaccinations for adults are not established. However, adult vaccination is extremely meaningful: increasing age means a higher susceptibility to infectious diseases, health problems and multimorbidity will increase. The burden of vaccine-preventable diseases is still high in Europe. Due to immunosenescence (older) adults are less protected against pathogens, antibody titers after vaccinations are lower and immunity lasts shorter. There is striking lack of data of adult vaccination rates and an international consensus regarding adult vaccination recommendations or guidelines are not available in Europe. In only six countries a comprehensive document describing recommended vaccinations for adults is available, among them Austria. The awareness of the importance of adult vaccination over the whole lifetime is not present to the necessary extent in Europe and has to be promoted.

Keywords Vaccination - Vaccinate - Adults · Ageing · Lifetime vaccination

\section{Einleitung}

Impfungen gehören weltweit zu den zehn erfolgreichsten Public Health Maßnahmen [1]. Der historische Vergleich von Daten vor und nach der Einführung von Impfprogrammen in den USA zeigt es deutlich: Reduktion von Diphtherie- und Poliofällen um $100 \%$, Hämophilus influenzae, Masern, Mumps, Röteln 99 \%, Tetanus 98 \%, Pertussis 93 \% oder Hepati- 
tis A um $91 \%$ und Hepatitis B um $83 \%$ [2]. Impfungen haben somit wesentlich zur Reduktion von Morbidität und Mortalität durch Infektionskrankheiten beigetragen, nach Angaben der Weltgesundheitsorganisation (WHO) retten Impfungen jedes Jahr ca. 2,5 Mio. Leben [3].

Mehr als ein halbes Jahrhundert nach der Einführung umfangreicher Impfprogramme treten in Europa immer noch zu viele Fälle impfpräventabler Erkrankungen (IPE) auf. Trotz insgesamt hoher Durchimpfungsraten bei vielen Infektionskrankheiten sind diese in einigen Subgruppen und Gemeinschaften immer noch unzureichend oder viel $\mathrm{zu}$ niedrig [4]. Der Import von IPE stellt ein besonderes Risiko für diese Gruppen dar und führt immer wieder zu Ausbrüchen mit teils erheblicher Morbidität und Mortalität, wie wir in den vergangenen Jahren in diversen Ländern wiederholt gesehen haben, u. a. Mumps (Irland, Niederlande, Moldawien, Deutschland und Tschechien), Masern (Frankreich, Spanien, Schweiz, Großbritannien, Deutschland, Kroatien und Bulgarien) oder Pertussis (Irland, Estland und Tschechien) (z. B. [5-7]). Zudem haben sich die am häufigsten betroffenen Altergruppen für traditionelle IPE geändert, Masern beispielsweise sind in vielen industrialisierten Ländern eine Infektion der Jugendlichen und Erwachsenen geworden [8]. Pertussis zeigt ebenso als Krankheit der Jugend und der Erwachsenen in vielen Ländern steigende Inzidenzen [9, 10].

Während bezüglich der Bedeutung von Impfungen für Säuglinge und Kinder ein breiter Konsens herrscht und weitreichende Impfprogramme installiert sind, ist das Konzept der Erwachsenenimpfung bei weitem nicht so gut etabliert. Die Strategie des lebenslangen Impfens steckt in den Kinderschuhen und muss in ganz Europa vorangebracht werden. Die vorliegende Publikation möchte dieses Konzept vorstellen, Hintergründe beleuchten und die Situation in Österreich, auch im europäischen Vergleich, aufzeigen.

\section{Tatsache Nr. 1: Die Menschen werden immer älter}

Die Lebenserwartung steigt um etwa 4 Monate jährlich an und ein heute in Österreich geborenes Mädchen hat gute Chancen, 100 Jahre alt zu werden. Die Zahlen für 2014 zeigen eine Lebenserwartung von 84 Jahren für weibliche und von 77 Jahren für männliche Neugeborene [11]. Eine rezente Studie errechnete die zukünftige, weiter steigende Lebenserwartung für 35 Industriestaaten, darunter auch für Österreich: über 81 Jahre für Männer und über 86 Jahre für Frauen im Jahr 2030 [12]. Ein weiterer wichtiger Parameter ist die verbleibende bzw. fernere Lebenserwartung, zum Beispiel haben heute 60-Jährige in Österreich noch 25 Jahre an Lebenserwartung vor sich. Der Anteil älterer Menschen steigt somit kontinuierlich an, ein Trend der weltweit $\mathrm{zu}$ beobachten ist und bereits 2050 werden weit über $30 \%$ der europäischen Bevölkerung $>60$ Jahre alt sein [13]. In Europa ist der
Anteil der älteren Menschen bereits heute weltweit am höchsten und dieser Trend wird sich die nächsten 5 Jahrzehnte fortsetzen [14].

Von 2015 bis 2030 wird die Zahl der Menschen $>60$ Jahre weltweit schätzungsweise um $56 \%$ ansteigen, von 901 Mio. heute auf 1,4 Mrd. und bis 2050 wird die globale Bevölkerung älterer Menschen mehr als die doppelte Größe von 2015 erreicht haben, nämlich fast 2,1 Mrd. [13]. Die Altersgruppe $>60$ Jahre wird dabei 3,5-mal schneller wachsen als die Gesamtpopulation [13], 2015 war einer von acht Menschen $>60$ Jahre alt, bis 2030 wird es einer von sechs und Mitte des 21. Jahrhunderts einer von fünf sein.

Die zunehmende Lebenserwartung wird einen Anstieg von unterschiedlichen Gesundheitsproblemen und Multimorbiditäten nach sich ziehen. Multimorbidität betrifft mehr als die Hälfte der älteren Bevölkerung und das Risiko steigt mit zunehmendem Alter, bei Frauen und mit sinkendem sozioökonomischen Status [15, 16]. Über $80 \%$ der >65-Jährigen leiden an einer chronischen Grunderkrankung, die per se eine Indikation für die Verabreichung bestimmter Impfungen darstellt (z. B. Influenza, Pneumokokken) [8]. Durchschnittlich sind drei bis neun chronische Krankheiten pro Person zu erwarten [17]. Typische altersassoziierte Erkrankungen werden ansteigen, wie z. B. Karzinome, kardiovaskuläre Erkrankungen, Diabetes, Adipositas oder Demenz, allesamt Erkrankungen, die Risikofaktoren für das Auftreten von IPE darstellen. Beispielsweise haben Menschen mit Diabetes ein erhöhtes Risiko für Influenza, Pneumokokkeninfektionen und für akute Hepatitis B, zudem kann die chronische Hepatitis schwerer verlaufen. Es besteht Evidenz für ein höheres Risiko für Herpes Zoster Erkrankungen und die postherpetische Neuralgie [18, 19].

Die steigende Lebenserwartung bringt es mit sich, dass einerseits eine zunehmende Zahl älterer Menschen in Institutionen wie Alters- oder Pflegeheimen lebt, was einen Risikofaktor für die Ansteckung mit bestimmten Krankheiten darstellt, andererseits haben immer mehr ältere Menschen ein hohes Gesundheitsbewusstsein, bleiben gesund und aktiv bis ins hohe Alter. Der Einsatz von Impfungen ist in beiden Lebenssituationen sinnvoll und wichtig, um Risiken $\mathrm{zu}$ minimieren bzw. gesundes Altern zur unterstützen [8].

\section{Tatsache Nr. 2: Immunoseneszenz - unser Immunsystem altert auch}

Mit zunehmendem Lebensalter kommt es $\mathrm{zu}$ weitreichenden Umgestaltungen sowohl im angeborenen als auch im adaptiven Immunsystem, wobei alle Ebenen von der hämatopoetischen Stammzelle bis zur reifen T- und B-Zelle betroffen sind. Diese Veränderungen werden auch als Immunoseneszenz bezeichnet und beeinflussen die Reaktionsfähigkeit auf Erreger und Impfstoffe gleichermaßen [20]. Beim angeborenen Immunsystem sind fast alle beteiligten Zellen 


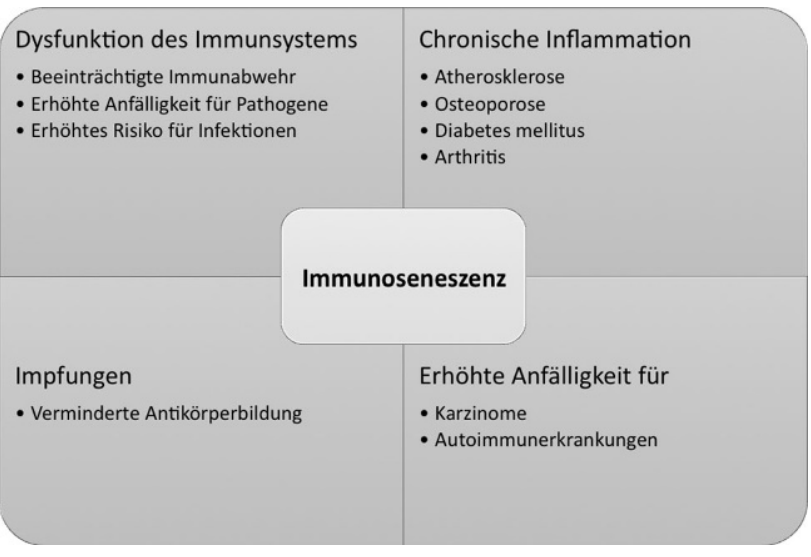

Abb. 1 Immunoseneszenz und potentielle Folgen. (Adaptiert nach [17])

betroffen, z. B. nimmt die Kapazität der neutrophilen Granulozyten und Makrophagen zur Produktion reaktiver Sauerstoffspezies oder die Fähigkeit zur Produktion chemotaktischer Botenstoffe ab. Die bereits im 2. Lebensjahrzehnt beginnende Involution des Thymus als dem zentralen Organ für die Reifung und Differenzierung von T-Zellen (Thymopoese) ist wohl die folgenreichste aller altersassoziierten Veränderungen im Immunsystem [21]. Damit einhergehend kommt es zu einer Abnahme zirkulierender naiver T-Zellen zugunsten hoch differenzierter Gedächtnis- und Effektorzellen, die unbekannte Antigene nicht mehr erkennen können. Diese Prozesse bedingen also ein eingeschränktes T-Zell-Rezeptor-Repertoire [20, 21]. Zusätzlich tritt eine Verminderung der T-Zell-Funktion auf, die sich u. a. durch eine verminderte Fähigkeit zur Zytokinproduktion und Proliferation auswirkt. Neben der Verschiebung innerhalb der T-Zellpopulation wirkt sich demnach auch die Alterung der T-Effektorzellen entsprechend aus. Die Fähigkeit zur Interaktion mit B-Zellen nimmt ebenfalls ab und die Folge ist eine gestörte Antikörperproduktion.

Die B-Zellpopulationen zeigen im Alter ähnliche Verschiebungen wie in der T-Zellpopulation, es kommt zu einer Abnahme naiver B-Zellen und einer Kumulation von B-Effektor-Zellen [22]. Nicht nur wird die Affinität zum jeweiligen Antigen geringer und die Antikörperantwort auf Krankheitserreger schwächer, auch die entsprechende Immunantwort auf Impfungen fällt schlechter aus. Die erzielten Antikörpertiter sind niedriger als bei jungen Menschen und die Immunität hält weniger lange an (,waning immunity“) [20]. Am Beispiel Tetanusimpfung wurde dies sehr gut belegt (mit verschiedenen Kombinationsimpfstoffen): jüngere Menschen hatten nach einer Boosterimpfung höhere Titer als Ältere, wobei sich dieser Effekt teilweise schon ab 40 Jahren zeigte und auch für andere Impfungen nachgewiesen werden konnte [23]. Eine Reihe weiterer Studien konnte dies ebenfalls demonstrieren [24-26]. In einer Studie mit Probanden zwischen 55 und 93 Jahren zeigte sich in der älteren Gruppe (65-93 Jahre) ein niedrigerer Tetanus-Antikörperspiegel als bei den Jüngeren. Die Höhe der Antikörperantwort war um so mehr verringert, je höher der Zeitabstand zur letzten Impfung war [27]. Eine Studie mit über 250 älteren Personen >60 Jahre kam zu dem Ergebnis, dass fünf Jahre nach einer Boosterimpfung gegen Diphtherie/Tetanus bei $45 \%$ die Diphtherieantikörper und bei $10 \%$ die Tetanusantikörper unter den protektiven Bereich gefallen waren [28]. Kürzere Boosterintervalle sind eine Möglichkeit, der „waning immunity“ entgegenzuwirken, allerdings besteht die Notwendigkeit der Entwicklung besser geeigneter Impfstoffe (näheres dazu in Tatsache Nr. 5: Vielzahl an Barrieren bei Erwachsenenimpfungen).

Selbstverständlich wirken sich die Umbauprozesse auch auf andere Bereiche aus, z. B. kommt es zu einer erhöhten Anfälligkeit für Krebs- oder Autoimmun- und chronische Erkrankungen, welche durch einen proinflammatorischen Status charakterisiert sind (z. B. Atherosklerose oder Diabetes mellitus) [29]. Abb. 1 zeigt einen vereinfachten Überblick über die wichtigsten Folgen der Immunoseneszenz.

Der Mensch ist infolge dieser umfassenden qualitativen Veränderungen mit zunehmendem Alter weniger gut gegen eindringende Pathogene geschützt und ein aufrechter Impfschutz somit von großer Bedeutung.

\section{Tatsache Nr. 3: Bedeutende Krankheitslast imprä- ventabler Erkrankungen bei Erwachsenen}

Infektionskrankheiten stellen immer noch die vierthäufigste Todesursache bei älteren Menschen in Industrieländern. Respiratorische Infektionen sind eine signifikante Ursache für Morbidität und Mortalität, zusammen repräsentieren sie die vierthäufigste Todesursache in entwickelten Ländern und betreffen vor allem Personen $>60$ Jahre $[29,30]$. In den USA beispielsweise sterben jedes Jahr ca. 45.000 Menschen an IPE, vor allem durch Influenza. Etwa 44.000 Fälle von invasiven Pneumokokkeninfektionen (IPD) mit 4500 Todesfällen bei Erwachsenen (>35 Jahre) wurden registriert. Über 4000 Todesfälle durch Humane Papilloma Viren (HPV)-assoziierte Zervixkarzinome traten trotz Screening auf [31]. Die Diagnose Zervixkarzinom wird in Österreich jährlich bei etwa 400 Frauen gestellt und kostet immer noch fast 200 Frauen das Leben [32]. In den Niederlanden war die Krankheitslast infolge von Infektionskrankheiten bei älteren Menschen durch Pneumokokkenerkrankungen (v. a. durch die hohe Mortalität) am höchsten, gefolgt von Influenza [33]. Infektionskrankheiten spielen eine bedeutende Rolle in der Krankheitslast älterer und gebrechlicher Menschen. Komorbiditäten und allgemeine Gebrechlichkeit erhöhen die Empfänglichkeit für viele Infektionskrankheiten, deren Inzidenz und Schweregrad mit zunehmendem Alter ansteigen und überdies zeigen ältere Menschen deutliche Besonderheiten in Bezug auf Klinik, Laborergebnisse, 
mikrobiotische Epidemiologie, Therapie und Infektionskontrolle [34, 35]. Laut einer niederländischen Studie wurden 2,6\% der Menschen >65 Jahre nach einer Pneumonie in ein Pflegeheim überstellt, was für einen erheblichen Abbau der physischen Kondition oder der Selbstständigkeit spricht [36]. Ältere Bewohner einer Langzeitpflegeeinrichtung zeigten nach dem Durchmachen einer Infektionskrankheit ein höheres Risiko für eine Abnahme des funktionellen Status [37].

In der Folge werden die wichtigsten Erkrankungen und dazugehörige Impfungen für (ältere) Erwachsene kurz beschrieben.

Herpes Zoster (HZ). Über $95 \%$ der Menschen $>50$ Jahre haben eine Immunität gegen Varizellen und tragen somit ein Risiko der Reaktivierung des Virus mit der Manifestation als Herpes Zostererkrankung [38]. Etwa 20-35\% der Bevölkerung in den Industrienationen werden einmal im Leben einen HZ entwickeln und dieses Risiko steigt mit zunehmendem Alter an (bis zu $50 \%$ bei >80-Jährigen). Die geschätzte Inzidenz liegt bei 3,4-4,82/1000 Personenjahre und steigt auf mehr als 11 bei den >80-Jährigen. In 13-40\% kommt es zu Komplikationen und außerdem gibt es einen Zusammenhang zwischen Inzidenz und Schweregrad der Erkrankung mit steigendem Alter [39]. Die postherpetische Neuralgie (PHN) ist die häufigste Komplikation und das Risiko, diese zu entwickeln, ist 4-mal höher für Personen $>70$ Jahre verglichen mit Personen jünger als 60 Jahre.

Seit einigen Jahren steht eine Lebendimpfung mit einer Effektivität in Bezug auf die Reduktion der Inzidenz von HZ und PHN bei Personen $>60$ Jahre um $51 \%$ bzw. 66 \% und einer Reduktion der Krankheitslast (zusammengefasste Messung aus Inzidenz, Dauer und Intensität der Schmerzen) um 61 \% zur Verfügung. Bei jüngeren Geimpften (50-59 Jahre) ist die Effektivität mit einer Reduktion der Inzidenz von HZ um $70 \%$ und der Krankheitslast um $73 \%$ um einiges besser [40, 41]. Somit profitieren vor allem jüngere Ältere von dieser Impfung im Sinne einer tatsächlichen Prävention der Erkrankung, bei älteren Menschen sind trotz einer insgesamt geringeren Wirksamkeit die Krankheitslast und Schwere der Erkrankung und im Besonderen die Lebensqualität erheblich verbessert.

Influenza. Die Influenza ist nicht nur eine der häufigsten, sondern auch eine der folgenschwersten Infektionskrankheiten. Weltweit erkranken jährlich 3-5 Mio. an der Influenza und 250.000-500.000 Menschen sterben [42]. Um die 40.000 Todesfälle treten während einer durchschnittlichen Epidemie in der Europäischen Union (EU) auf und in Österreich werden etwa 350.000-400.000 Erkrankungsfälle und 1000-1200 Todesfälle verzeichnet [43-45]. Die Influenza gehört europaweit zu den am meisten unterschätzten Infektionskrankheiten und die Impfung zu einer der am schlechtesten genutzten Präventionsmaßnahmen überhaupt. Mit einer Durchimpfungsra- te von nur ca. $6 \%$ der Bevölkerung gehört Österreich zu den Ländern mit den niedrigsten Impfraten [46]. Sowohl ein Großteil der Bevölkerung als auch erhebliche Teile des Gesundheitspersonals begegnen der Krankheit Influenza und der Impfung mit erheblicher Ignoranz, ungeachtet der Tatsache, dass Österreich zu den Ländern mit den umfassendsten Impfempfehlungen weltweit gehört [47].

Es gibt eine Vielzahl von Influenzaimpfstoffen (Spalt-, Subunit- und Ganzvirusimpfstoffe, intranasale Lebendimpfstoffe, trivalent, quadrivalent - ab der Saison 2017/2018 auch in Österreich erhältlich -, Impfstoffe für Kinder, Erwachsene und ältere Menschen). Da ein umfassender Einblick in diese komplizierte Thematik im Rahmen dieser Publikation unmöglich ist, sei an dieser Stelle ganz kurz zusammengefasst: Wenn die Impfstoffstämme gut mit den zirkulierenden Stämmen übereinstimmen, liegt die Wirksamkeit der Impfung bei <65-Jährigen bei 70-90 \% (je jünger, desto besser), bei älteren Menschen ist die Wirksamkeit in der Regel deutlich geringer, der Schutz vor influenzabedingten Komplikationen liegt aber deutlich höher [48].

Masern. Die mehrfach verschobene, für 2015 geplante Ausrottung der Masern ist wieder in weite Ferne gerückt. Aufgrund zahlreicher großer Ausbrüche verdoppelte sich die Zahl der Masernfälle mit $>30.000$ im Jahr 2015 in Europa im Vergleich zum Vorjahr. Über $43 \%$ der Fälle waren Erwachsene $>20$ Jahre [49]. Die Masernepidemie läuft auch 2016 und 2017 weiter, wobei 2017 ein ganz besonderes starkes Jahr zu werden scheint. Zwischen April 2016 und März 2017 wurden von 30 EU/EFTA-Ländern fast 6600 Fälle gemeldet, 26 Staaten verzeichneten dabei durchgehend das ganze Jahr Fälle [50]. Alleine in den ersten drei Monaten des Jahres 2017 wurden 2480 Fälle beobachtet, 2016 waren es in diesem Zeitraum hingegen „nur“ 530 gewesen. Am stärksten betroffen waren Rumänien (750), Italien (>680) und Deutschland (>400). Österreich gehört 2017 zu den elf Ländern, in denen während der ersten drei Monate mehr Fälle registriert wurden als im gesamten Jahr zuvor (Stand 08.06.2017: 78 Fälle) [51]. Hauptursache für die hohen Fallzahlen sind niedrige Durchimpfungsraten in einigen europäischen Ländern. Die zweimalige MMR-Lebendimpfung muss in $95 \%$ der Bevölkerung erreicht werden, um das Virus auszurotten, was in vielen Regionen nicht nachhaltig gelingt. In Österreich und einigen anderen europäischen Ländern (z. B. Deutschland, Kroatien, Italien, Rumänien) gibt es große Impflücken vor allem bei Kindern und jungen Erwachsenen, die oftmals nur eine Impfung erhalten haben. In Österreich gibt es Impflücken vor allem bei Erwachsenen geboren in den 1990er-Jahren, zudem ist ein Drittel der 15- bis 30-Jährigen (das ist > eine halbe Million) nur einmal geimpft [51]. Bei (älteren) Erwachsene fehlt mitunter der Schutz gegen Masern, da sie zu alt waren, als die Impfung in den 1980er-Jahren einge- 
führt worden war oder sie die Impfung aus anderen Gründen versäumt hatten [52].

Bei der Masernimpfung handelt es sich um eine Lebendimpfung, die im Rahmen einer Kombinationsimpfung mit Mumps und Röteln verabreicht wird (MMR-Impfung).

Pertussis. Die Einschätzung von Pertussis als Kinderkrankheit ist ein großes Missverständnis. Die Krankheitslast bei Jugendlichen und Erwachsenen wächst kontinuierlich in Europa [9]. Laut Angaben der WHO gab es weltweit im Jahr 2015 über 142.000 Fälle und fast 90.000 Todesfälle [53]. Das European Center for Disease Control (ECDC) verzeichnete 2014 fast 41.000 Fälle in 29 EU/EEA-Ländern [10]. In Österreich werden 700-1000 Fälle pro Jahr gemeldet. Bei einer geschätzten Inzidenz von 130/100.000 kann man eine Fallzahl von etwa 10.000 Erkrankungen annehmen [54]. Die Krankheit verläuft bei Jugendlichen und Erwachsenen anders als bei den Kleinsten, wobei ein über Wochen anhaltender Husten das Hauptsymptom ist, bei dem oftmals nicht an Pertussis gedacht wird. Somit stellen diese Personen, ohne es zu wissen, eine wesentliche Ansteckungsquelle für die am meisten gefährdeten - Säuglinge $<1 \mathrm{Jahr}-$ dar [9].

Die Impfung wird bei Erwachsenen im Rahmen von Kombinationsimpfstoffen verabreicht (mit Diphtherie und Tetanus als 3-fach oder zusätzlich mit Polio als 4 -fach Kombination).

Pneumokokken. Nach der Einführung der konjugierten Impfstoffe in viele Kinderimpfprogramme kam es in diesen Ländern, bedingt durch den Herdeneffekt, zu einem teilweise dramatischen Absinken von IPDs auch bei Erwachsenen [55]. Dennoch ist die Krankheitslast durch Pneumokokkeninfektionen vor allem bei älteren Erwachsenen immer noch hoch (nicht invasive Pneumokokkenerkrankungen, im besonderen Pneumonie, und invasive Formen). Nach Angaben des ECDC lag die Inzidenz in Europa im Jahr 2014 zwischen 0,4/100.000 und 20/100.000 und mehr als 17.000 Fälle wurden von 28 Ländern gemeldet (wobei unterschiedliche Überwachungsstrategien einen Vergleich schwierig machen). Die großen Unterschiede in den gemeldeten Inzidenzraten reflektieren sowohl echte Differenzen als auch Verschiedenheiten bei Diagnostik und Überwachung [56]. IPDs liegen in Österreich bei ca. 300 Fällen pro Jahr (2014: 323, 2015: 422; Inzidenz 2014: 3,8 und 2015: 4,92), die Inzidenz steigt ab dem 45. Lebensjahr kontinuierlich an und ist in der Regel bei den >80-Jährigen am höchsten (2014: 15 und 2015: 14,9) [57].

Die Polysaccharidimpfstoffe (PPV23) sind seit langem im Einsatz und haben sich im Rahmen ihrer Möglichkeiten mit einem protektiven Effekt gegen IPD und Pneumonie aller Ursachen bei gesunden jungen Erwachsenen und zu einem geringeren Ausmaß beim Schutz gegen IPD bei Älteren $>65$ Jahre durchaus bewährt. Beobachtungsstudien haben eine
Wirksamkeit zwischen 50 und $80 \%$ gegen IPD bei älteren Erwachsenen und immunkompetenten Personen mit diversen Grunderkrankungen gezeigt [58]. Konjugierte Pneumokokkenimpfstoffe bewirken eine bedeutende Risikoreduktion für Impfstoff-spezifische Pneumokokkenerkrankungen (mit einer relativen Risikoreduktion zwischen 20 und $75 \%$ ) und eine geringere Reduktion für Infektionskrankheiten anderer Ursache. Insgesamt zeigen Studien trotz unterschiedlicher Populationen und Settings relativ konsistente Ergebnisse [59].

Polio. Europa wurde 2002 für poliofrei erklärt, es gibt keine Zirkulation des Wildvirus. Allerdings bleibt das Risiko eines Imports in einigen Ländern hoch. Beispielsweise wurden 2013 Wildtyp-Zirkulationen in Israel entdeckt und es gab Ausbrüche von paralytischer Polio in Syrien und in Teilen des Horns von Afrika, was das Risiko eines Imports in die EU/EEA erhöht [60]. Der orale Polioimpfstoff (OPV) wurde in der Vergangenheit während großer Ausrottungskampagnen und wird heute noch in endemischen Ländern eingesetzt, da er eine intestinale Immunität induziert, günstig und leicht anwendbar ist. In den europäischen Ländern wird ausschließlich (Ausnahme Polen) der inaktivierte Totimpfstoff (IPV) verwendet, da hier das Risiko einer Impfstoff-assoziierten Polio nicht besteht (OPV 4:1.000.000 Geimpften und ungeimpften Kontakten).

Solange es Regionen mit endemischer Polio gibt (derzeit Nigeria, Pakistan, Afghanistan) und es in ehemals poliofreien Ländern immer wieder durch Einschleppung zu Ausbrüchen kommt, muss in der EU solange weiter geimpft werden, bis das Poliovirus endgültig ausgerottet ist. Nach Schätzungen des ECDC sind 12 Mio. EU-Bürger jünger als 30 Jahre nicht gegen Polio geimpft [61].

Die Impfung wird bei Erwachsenen im Rahmen von Kombinationsimpfstoffen verabreicht (mit Diphtherie und Tetanus als 3-fach oder zusätzlich mit Pertussis als 4-fach Kombination) und es gibt auch einen Einzelimpfstoff.

Tetanus/Diphtherie. Die Inzidenzen von Diphtherie und Tetanus sind in Europa sehr niedrig, mit 161 Tetanus Fällen und 36 Fällen von Diphtherie pro Jahr (Durchschnitt 2009-2014) [62]. Sowohl Tetanus- als auch Diphtherieantikörpertiter bei Erwachsenen und speziell bei Älteren sind häufig unter dem notwendigen Seroprotektionslevel, wie diverse Studien aus unterschiedlichen Ländern belegen [4, 62]. Daten aus Österreich zeigen, dass $12 \%$ der Studienpopulation $>65$ Jahre nicht ausreichend gegen Tetanus und sogar $65 \%$ nicht gegen Diphtherie geschützt waren [28]. Eine weitere Studie in 6 europäischen Ländern und Israel zeigte auf, dass zunehmendes Alter mit einem Ansteigen der seronegativen Personen einhergeht, da keine Boosterimpfungen durchgeführt wurden [4, 63]. Auch wenn die Erkrankungszahlen in Europa sehr 
Tab. 1 Bandbreite der Impfempfehlungen für Erwachsene in Europa. (Adaptiert nach [66])

\begin{tabular}{|l|l|}
\hline Art der Empfehlungen & Europäische Länder \\
\hline Umfassendes Dokument oder Impfplan & Deutschland, Frankreich, Großbritannien, Island, Österreich, Spanien \\
\hline Plan mit spezifischen Empfehlungen für wenigstens eine Impfung & $\begin{array}{l}\text { Bulgarien, Dänemark, Estland, Finnland, Griechenland, Lettland, Luxemburg, } \\
\text { Malta, Niederlande, Portugal, Rumänien, Slowakei, Slowenien, Tschechische } \\
\text { Republik, Ungarn, Zypern }\end{array}$ \\
\hline Kein spezifischer Plan oder Dokument vorhanden & Belgien, Irland, Italien, Litauen, Polen, Schweden
\end{tabular}

niedrig sind, müssen die Impfraten gegen Diphtherie weiterhin auf hohem Niveau gehalten werden, da diese Krankheit immer noch endemisch in Asien, Afrika und Südamerika vorkommt und somit Importe jederzeit möglich sind.

Die Impfung wird in der Regel im Rahmen von diversen Kombinationsimpfstoffen verabreicht.

\section{Tatsache Nr. 4: Niedrige Impfraten und} mangelnde Impfempfehlungen in Europa

Leider gibt es in Europa einen eklatanten Mangel an Daten zu Durchimpfungsraten bei Erwachsenen und zudem keinen internationalen Konsensus bezüglich Impfempfehlungen oder Richtlinien für Erwachsene. Die Unterschiede in den jeweiligen nationalen Impfempfehlungen und auch in der Umsetzung dieser sind groß, sei es im Hinblick auf die gesetzten Prioritäten, die entsprechenden Budgets und das Impfservice bzw. vorhandene Infrastrukturen [64]. Kein einziges EU/ EFTA Land registriert routinemäßig die Impfraten für empfohlene nationale Impfungen bei Erwachsenen, mit Ausnahme von Influenza (20/29 Ländern), Hepatitis B (23/29) und Tetanus/Diphtherie-Impfung (6/29) [65-67]. Lediglich die Influenza Impfraten werden seit 2007 durch das ECDC jährlich erhoben [68, 69]. Erschwerend kommt hinzu, dass die Erhebungsmethoden bez. Impfraten oder auch die Meldung von Nebenwirkungen europaweit ebenfalls sehr unterschiedlich gehandhabt werden $[64,69]$. Es gibt Bestrebungen des ECDC - durch ein im Rahmen des VENICE III Progamms (Vaccine European New Integrated Collaboration Effort) gestartetes Projekt, das European VAccination COverage Collection System (EVACO) - verlässliche Daten zu Impfraten in Europa zur Verfügung zu stellen [68].

Viele europäische Länder haben unzureichende oder gar keine Impfempfehlungen für Erwachsene. Nur sechs Länder verfügen über ein ausführliches Dokument, das alle Impfungen beschreibt, die für Erwachsene empfohlen sind, darunter auch Österreich (Tab. 1). Auffallend ist, dass diese Länder auch die höchste Anzahl an Impfungen empfehlen [65, 66]. In der Mehrheit der europäischen Länder sind die meisten Impfungen für Erwachsene nur für spezifische Risikogruppen empfohlen. Die am häufigsten empfohlenen Impfungen für Erwachsene in 31 hochentwickelten Volkswirtschaften waren Impfungen gegen Influenza, Tetanus, Diphtherie, Pneumokokken und Hepatitis B [67].
Außer der Vorgabe von WHO und ECDC, dass bis zur Saison 2014/2015 $75 \%$ aller >65-Jährigen gegen Influenza geimpft sein sollten, gibt es keine weiteren definierten Ziele in Europa [66]. Diese von WHO und ECDC empfohlene Influenza Durchimpfungsrate wurde bislang von nur wenigen Ländern erreicht [70]. Zu den Ländern, die in der Saison 2014/2015 dem Ziel nahe waren, gehörten Großbritannien (73\%) und die Niederlande (67\%) und zu den Ländern mit vergleichsweise hohen Durchimpfungsraten zählten Irland (60\%), Spanien (56\%), Italien (49\%) und Frankreich $(48 \%)$ [71]. Österreich steht im europäischen Vergleich mit einer Impfrate von ca. $30 \%$ bei den $>60$ Jährigen in der Primärversorgung ziemlich schlecht da (2010/2011) [72].

Es gibt Initiativen, die diese unzureichende Situation bei Erwachsenenimpfungen ändern möchten und sich für entsprechende harmonisierte Empfehlungen in Europa einsetzen, z. B. von großen Gesellschaften wie der European Federation of Internal Medicine, der European Union Geriatric Medicine Society und der International Association of Gerontology and Geriatrics-European Region, oder der Escmid Vaccine Study Group, der European Geriatric Medicine Society (EUGMS) und der World Association for Infectious Diseases and Immunological Disorders [66, 73, 74].

Impfungen in Europa bedienen im Großen und Ganzen fünf Gruppen von Erwachsenen: Personen, die Boosterimpfungen benötigen (um den Impfschutz aufrecht $\mathrm{zu}$ erhalten), ältere Menschen >60 bzw. > 65 Jahre, Risikogruppen mit Komorbiditäten, Hochrisikogruppen wie Schwangere oder Gesundheitspersonal und Reisende. Gesunde Erwachsene bleiben als wichtige Zielgruppe für Impfungen oftmals unbeachtet. Dabei gibt es sehr gute Gründe für einen umfassenden, aufrechten Impfschutz in dieser Gruppe, zumal gesunde Erwachsene 50-60\% der europäischen Bevölkerung stellen und somit schon rein zahlenmäßig von erheblicher Bedeutung sind, im Hinblick z. B. auf Erwerbstätigkeit, Krankheitslast oder als potentielle Überträger.

\section{Tatsache Nr. 5: Vielzahl an Barrieren bei Erwachsenenimpfungen}

Die Liste an Hürden und Hindernissen, die der Impfung von Erwachsenen entgegenstehen, ist lang: von logistischen Problemen, mangelndem Bewusstsein bei den Betroffenen und dem Gesundheitspersonal, einem Mangel an korrekter Datenerfassung und com- 
Tab. 2 Häufige Gründe für niedrige Impfraten bei Erwachsenen, geordnet nach betroffenen Gruppen. (Adaptiert nach [75, 76, 87])

\begin{tabular}{|c|c|c|c|}
\hline Gründe für niedrige Impfraten & Erwachsene & Gesundheitspersonal & Gesundheitswesen \\
\hline Gesundheitsbewusstsein/verhalten & $x$ & - & - \\
\hline Unterschätzen von Wichtigkeit \& Krankheitslast der IPE & $\mathrm{x}$ & $x$ & - \\
\hline $\begin{array}{l}\text { Mangel an Wissen \& Bewusstsein um die Notwendigkeit, Indikationen und den } \\
\text { Wert von Impfungen für Erwachsene }\end{array}$ & $\mathrm{x}$ & $\mathrm{x}$ & $\mathrm{x}$ \\
\hline $\begin{array}{l}\text { Unsicherheit oder Mangel an Wissen über Inhaltsstoffe, Sicherheit \& Effektivi- } \\
\text { tät von Impfungen für Erwachsene }\end{array}$ & $x$ & $x$ & - \\
\hline Ungenügende Aktivierung hinsichtlich des Themas (wie bei Kinderimpfungen) & - & $x$ & $x$ \\
\hline Mangel an Impfempfehlungen & - & $x$ & $\mathrm{x}$ \\
\hline Versäumte Gelegenheiten bei Systemkontakt & - & $x$ & $x$ \\
\hline Negativer Einfluss durch Familie \& Freunde & $\mathrm{x}$ & - & - \\
\hline Verwirrung durch unterschiedliche Impfpläne für diverse Gruppen & - & $\mathrm{x}$ & $\mathrm{x}$ \\
\hline Keine routinemäßige Erfassung des Impfstatus & - & $x$ & $x$ \\
\hline Mangel an Zeit für Information \& Aufklärung & - & $\mathrm{x}$ & - \\
\hline Kulturelle und sprachliche Probleme & $\mathrm{x}$ & $x$ & - \\
\hline $\begin{array}{l}\text { Fehlen einer zentralen „Führerschaft“ (Persönlichkeit oder Organisation); } \\
\text { fehlendes medizinisches „Zuhause“ des Themas }\end{array}$ & - & $\mathrm{x}$ & $x$ \\
\hline Mangel an Unterstützung durch medizinische Gesellschaften & - & $\mathrm{x}$ & - \\
\hline Mangel an Finanzierung \& Bezahlung & $\mathrm{x}$ & $\mathrm{x}$ & $\mathrm{x}$ \\
\hline Diverse logistische Probleme & $\mathrm{x}$ & $x$ & $x$ \\
\hline
\end{tabular}

putergestützten Impfregistern, fehlender Routine bei Überprüfung des Impfstatus, Fehlen von koordinierten Impfprogrammen für Erwachsenimpfungen oder zu wenig Zeit für die Aufklärung über Risiken und Nutzen von Impfungen sind die Herausforderungen enorm [65, 75]. Ein systematischer Review identifizierte sechs große Bereiche, die die Impfwilligkeit von Erwachsenen beeinflussen:

1. generelle Einstellungen und Überzeugungen (positive und negative),

2. wahrgenommenes Risiko bzw. Schweregrad von Krankheiten (Wissen, wahrgenommene Anfälligkeit, eigene Erfahrungen),

3. Impfungsbezogene Faktoren (Nebenwirkungen, Effektivität, Meinung zu Inhaltsstoffen, Wissen),

4. Empfehlung und Information (Einfluss durch Gesundheitspersonal, Verwandte und andere, Informationsquelle bzw. format)

5. allgemeines Gesundheitsverhalten inklusive bisherige Impfungen und anderes präventives Verhalten und

6. Zugänglichkeit und Leistbarkeit (logistische Gründe, Kombination von Impfungen und Kosten) [76]. Tab. 2 zeigt einen Überblick über die wesentlichen Barrieren bei Erwachsenenimpfungen und versucht eine Einordnung der Gründe nach betroffen Gruppen.

$\mathrm{Zu}$ diesen zahlreichen Problemen kommt erschwerend hinzu, dass die Wirksamkeit mancher Impfungen für Erwachsene unzureichend ist. Hier soll beispielhaft die Influenzaimpfung genannt werden, die gerade in der Gruppe mit dem höchsten Risiko für schwere Verläufe und Komplikationen, den >65-Jährigen, eine nur moderate Wirksamkeit zeigt (dabei dennoch eine Reduzierung der Hospitalisierungen und der Gesamtmortalität bewirkt, [48]). An der Entwicklung effizienterer Impfstoffe - z. B. durch Verwendung spezifischer Adjuvantien, höherer Antigendosierungen oder alternativer Applikationswege - wird seit Jahren gearbeitet [77].

In diesem Zusammenhang kommt dem Ansatz, durch Erhöhung der Impfraten bei Kindern die Älteren durch die Entstehung einer Herdenimmunität zu schützen, eine immer größere Bedeutung zu. Ein möglicher Herdeneffekt durch die Influenzaimpfung von Kindern wurde bereits in etlichen Studien nachgewiesen [78-83]. Beispielsweise zeigte eine kanadische Studie einen dramatischen Herdeneffekt durch die Impfung von Schulkindern und Jugendlichen mit einer 61 \% Effektivität in der Reduktion von laborbestätigter Influenza bei nicht geimpften Erwachsenen [82]. Nach der Einführung der konjugierten Pneumokokken-Impfstoffe (PNC 7, 10 und 13 valent) in die Kinderimpfprogramme kam es in vielen Ländern $\mathrm{zu}$ einem dramatischen und raschen Absinken von IPD bei den geimpften Kindern, aber - bedingt durch den Herdeneffekt - auch in anderen Altersgruppen (z. B. [84, 85]). In den USA beispielsweise kam es 3 Jahre nach der Einführung des 13-valenten PNC zu einem schnellen Rückgang von allen IPD bei (nicht geimpften) Erwachsenen um 12-32\% und von durch Impfstoff-Serotypen verursachten IPD um 58-72\% [84].

Ein weiteres Beispiel für die suboptimale Effektivität von Impfstoffen bei älteren Erwachsenen ist, wie schon beschrieben wurde, die Impfung gegen Herpes Zoster [40]. Ein derzeit in Phase 3 befindlicher Tot- 
Tab. 3 Österreichische Impfempfehlungen für Erwachsene mit kompletter Grundimmunisierung. (Adaptiert nach [47])

\begin{tabular}{|c|c|c|c|}
\hline $\begin{array}{l}\text { Alter } \Rightarrow \\
\text { Impfung } \Downarrow\end{array}$ & $16-18$ & $51-60$ & Ab 61 \\
\hline $\begin{array}{l}\text { Diphtherie (dip) } \\
\text { Tetanus (TET) } \\
\text { Pertussis (PEA) } \\
\text { Poliomyelitis } \\
\text { (IPV) }\end{array}$ & \multicolumn{2}{|l|}{ Booster alle 10 Jahre } & Booster alle 5 Jahre \\
\hline Humane Papillom-Viren (HPV) & Nachholimpfung ${ }^{\mathrm{a}}$ & - & \\
\hline $\begin{array}{l}\text { Mumps } \\
\text { Masern (MMR) } \\
\text { Röteln }\end{array}$ & \multicolumn{2}{|l|}{ Nachholimpfung ${ }^{b}$} & \\
\hline FSME & \multicolumn{2}{|l|}{ Booster alle 5 Jahre } & Booster alle 3 Jahre \\
\hline Pneumokokken & - & 13 valent $\Rightarrow 23$ valent $^{c}$ & \\
\hline Herpes Zoster (HZV) & - & Einmalige Impfung & \\
\hline Influenza & \multicolumn{2}{|l|}{ Jährlich } & \\
\hline
\end{tabular}

impfstoff verspricht mit einer Wirksamkeit von über $97 \%$ einen erheblich verbesserten Schutz im Vergleich zum Lebendimpfstoff und das in allen Altersgruppen (50-59, 60-69 und $\geq 70$ ) [86].

\section{Impfen über die gesamte Lebenszeit}

Nachdem Impfen lange Zeit vor allem als Kindersache angesehen wurde, gewinnt die Erkenntnis der Notwendigkeit eines lebenslangen Impfens zunehmend an Bedeutung. In den USA geschieht dies schon seit längerem, in Europa erst seit wenigen Jahren. In den letzten Jahren war eine zunehmende Zahl an Publikationen $\mathrm{zu}$ verzeichnen, die sich der Bedeutung von Impfungen für Erwachsene bzw. ältere Personen annahmen [8, 21, 65-67, 73-76, 88]. Alle diese Publikationen kommen im Grunde zu demselben Schluss, dass Impfungen auch für Erwachsene ein entscheidender Teil in der Prävention von Krankheiten und für den Erhalt von Gesundheit im Sinne des „gesunden Alterns" sind und die Zukunft des Impfens im Grunde das lebenslange Impfen ist. Eine Impfstrategie von der frühesten Kindheit über die gesamte Lebenszeit ist notwendig.

Zusammenfassend sind Impfungen für Erwachsene aus verschiedenen Gründen wichtig und sinnvoll:

1. sie haben möglicherweise in der Kindheit nicht alle Impfungen erhalten, auch weil immer wieder Impfungen in nationale Routine-Impfpläne aufgenommen wurden. Viele Erwachsene sind somit immer noch ungeschützt gegen eine oder mehrere IPE;

2. neue Impfstoffe speziell für Erwachsene sind heutzutage vorhanden, z. B. der Herpes Zoster Impfstoff;

3. aufgrund der Immunoseneszenz lässt die in der Jugend erworbene Immunität nach;

4. ältere Erwachsene oder chronisch Kranke sind empfänglicher für diverse IPE [8].

\section{Impfempfehlungen für Erwachsene in Österreich}

Wie bereits erwähnt gehört Österreich zu den wenigen Ländern in Europa, die umfassende Impfempfehlungen für Erwachsene formuliert haben. Diese Empfehlungen werden im österreichischen Impfplan beschrieben und jährlich aktualisiert [47]. Tab. 3 zeigt einen Überblick über die empfohlenen Impfungen. Zudem gibt es ein Supplement zum allgemeinen Impfplan, das sich mit dem Thema „Impfungen im erwerbsfähigem Erwachsenenalter" auseinandersetzt und ebenfalls detaillierte Empfehlungen auflistet [88]. In Österreich besteht keine Impfpflicht, eine Diskussion darüber ist angesichts immer wieder auftretender Masernepidemien angezeigt und scheint langsam in Gang zu kommen (z. B. [89]).

Die österreichischen Empfehlungen für Erwachsene sind sehr umfangreich und beinhalten 12 Impfungen, die meisten davon sind Boosterimpfungen (nach abgeschlossener Grundimmunisierung zumeist im Kindes- und Jugendalter), Pneumokokken und Herpes Zoster Impfung sind klassische „Altersimpfungen" für Personen $>50$ Jahre. Die österreichischen Empfehlungen beinhalten einige Besonderheiten: z. B. ist die Influenza-Impfung bereits ab 50 Jahren generell empfohlen, in den allermeisten Ländern gilt diese Empfehlung erst ab 65 oder 60 Jahren. Zudem sind kürzere Abstände zwischen den Boosterimpfungen ab 60 Jahren empfohlen, bei der 4-fach Impfung (Dip/TET/PER/IPV) alle 5 Jahre (statt alle 10 Jahre bei Personen <60) und bei der FSME-Impfung alle 3 Jahre (statt alle 5 Jahre bei Personen <60). Dieses Zugeständnis an das alternde Immunsystem findet sich in kaum einem anderen Land.

Des Weiteren behandelt der österreichische Impfplan in eigenen Kapiteln Empfehlungen für besondere erwachsene Personengruppen, z. B. Impfungen bei Kinderwunsch, in der Schwangerschaft und Still- 
zeit, bei Personen mit Immundefekten oder für Personal des Gesundheitswesens (für diese Gruppe gibt es wiederum als Ergänzung zum Impfplan eine weiterführende Publikation „Impfungen für MitarbeiterInnen des Gesundheitswesen“ [90]).

Insgesamt stehen somit in Österreich sehr gute Impfempfehlungen für Erwachsene zur Verfügung, allerdings ist die entsprechende Umsetzung in einigen Bereichen noch mehr als ausbaufähig.

\section{Ausblick}

Um die Situation in Österreich (und auch in Europa) nachhaltig $\mathrm{zu}$ verbessern, ist ein Paradigmenwechsel nötig. Nur mit einem koordinierten umfassenden Impfkonzept für Erwachsene können wesentliche Veränderungen in Gang gesetzt werden [87]. Man könnte sich zu Beginn auf drei wesentliche Bereiche konzentrieren:

1. Das teilweise dramatische Unterschätzen der Gefahren und Risiken durch IPD und des Wertes von Impfungen im Erwachsenenalter - vor allem auch im Hinblick auf das gesunde Altern - muss thematisiert werden. Entsprechende Aufklärung der Bevölkerung und Ausbildung des Gesundheitspersonals sind dabei essentielle erste Schritte. Der Wert einer Impfempfehlung durch einen vertrauten Arzt oder Ärztin kann nicht hoch genug geschätzt werden. Halbwahrheiten und Unwissen bezüglich der Wirksamkeit und Sicherheit von Impfstoffen sind wichtige Aspekte, die angesprochen werden müssen.

2. Ein weiterer bedeutender Punkt wäre die Kostenübernahme für die wichtigsten Impfungen im Erwachsenenalter durch den Staat, ähnlich dem Kinderimpfkonzept, in dem bestimmte empfohlene Impfungen für alle Kinder und Jugendlichen bis 15 Jahre gratis zur Verfügung gestellt werden (in Österreich ist nur die MMR-Impfung für alle Erwachsenen kostenfrei). Die Finanzierung zumindest einiger Impfungen wäre auch als wichtiges Signal an die Menschen zu sehen, dass dem Staat der Erhalt der Gesundheit durch den Einsatz einer der erfolgreichsten präventiven Maßnahmen, den Impfungen, wichtig ist. Ein weiterer Aspekt ist natürlich, die Menschen entsprechend finanziell zu entlasten und die damit verbundene Hoffnung, die Impfwilligkeit zu erhöhen.

3. Die Verbesserung der Nutzung von Systemkontakten ist eine Maßnahme, die relativ schnell umgesetzt werden könnte. Die Forderung der WHO jeden Systemkontakt, auch im Rahmen von Spitalsaufenthalten, für die Überprüfung des Impfstatus, Aufklärung und Durchführung von fehlenden Impfungen zu nützen, ist sehr sinnvoll [47]. Konzepte für eine möglichst rasche und effektive Umsetzung sollten von allen Beteiligten gemeinsam entwickelt werden.

\section{Schlussfolgerungen}

Impfungen gehören zu den effektivsten Maßnahmen in der Prävention und haben in der Vergangenheit unzählige Leben gerettet und Leid verhindert. Die Erkenntnis, dass Impfungen nicht nur Kindersache, sondern auch für Erwachsene jeden Alters wichtig und sinnvoll sind, ist zwar nicht wirklich neu, das Bewusstsein um die Wichtigkeit von Impfungen über die gesamte Lebensspanne ist vor allem in Europa noch nicht in dem Maße vorhanden, wie es nötig wäre. Eine Verbesserung der Impfraten bei Erwachsenen würde Morbidität und Mortalität zahlreicher Krankheiten reduzieren, damit auch viele Hospitalisierungen verhindern und hohe Kosten senken. Ein weiterer wichtiger Aspekt, vielleicht sogar der Wichtigste, ist die Verbesserung der Lebensqualität vor allem für ältere Personen, die durch schwere Verläufe von Infektionskrankheiten besonders gefährdet sind. Die Entwicklung von effizienten Impfstoffen gerade für ältere Erwachsene hat höchste Priorität. Österreich gehört mit seinen Impfempfehlungen für Erwachsene zu den Vorreitern in Europa und ist damit auf einem guten Weg. Es müssen allerdings noch große Anstrengungen unternommen werden, um diese Empfehlungen in der Realität umsetzen zu können und die Impfraten zu erhöhen.

Acknowledgements Open access funding provided by Medical University of Vienna.

\section{Einhaltung ethischer Richtlinien}

Interessenkonflikt U. Kunze und E. Groman geben an, dass kein Interessenkonflikt besteht.

Open Access Dieser Artikel wird unter der Creative Commons Namensnennung 4.0 International Lizenz (http:// creativecommons.org/licenses/by/4.0/deed.de) veröffentlicht, welche die Nutzung, Vervielfältigung, Bearbeitung, Verbreitung und Wiedergabe in jeglichem Medium und Format erlaubt, sofern Sie den/die ursprünglichen Autor(en) und die Quelle ordnungsgemäß nennen, einen Link zur Creative Commons Lizenz beifügen und angeben, ob Änderungen vorgenommen wurden.

\section{Literatur}

1. Centers for Disease Control and Prevention (CDC). Ten great public health achievements - worldwide, 2001-2010. MMWR Morb Mortal Wkly Rep. 2011;60:814-8.

2. Roush SW, Murphy TV. Vaccine-preventable disease table working group. Historical comparisons of morbidity and mortality for vaccine-preventable diseases in the united states. JAMA. 2007;298:2155-63.

3. World Health Organization, UNICEF, World Bank. State of the world's vaccines and immunization. 3. Aufl. Geneva: World Health Organization; 2009.

4. Wicker S, Maltezou HC. Vaccine-preventable diseases in Europe: where do we stand? Expert Rev Vaccines. 2014; https://doi.org/10.1586/14760584.2014.933077.

5. Otto W, Mankertz A, Santibanez S, et al. Ongoing outbreak of mumps affecting adolescents and young adults in 
Bavaria, Germany, August to October 2010. Euro Surveill. 2010;15:21-4.

6. Monsel G, Rapp C, Duong TA, et al. Measles in adults: an emerging disease not sparing medical staff. Ann Dermatol Venereol.2011;138:107-10.

7. Barret AS, Ryan A, Breslin A, et al. Pertussis outbreak in northwest Ireland, January-June 2010. Euro Surveill. 2010;15:3-7.

8. Bonanni C, Donato SR, et al. Lifelong vaccination as a key disease-prevention strategy. Clin Microbiol Infect. 2014;20:32-6.

9. Zepp F, Heininger U, Mertsola J, et al. Rationale for pertussis boostervaccination throughoutlifein Europe. Lancet Infect Dis. 2011;11:557-70.

10. European Centre for Disease Prevention and Control. Annual Epidemiological Report 2016 - Pertussis. [Internet]. Stockholm: ECDC. 2016. http://ecdc.europa.eu/en/ healthtopics/Pertussis/Pages/Annual-epidemiologicalreport-2016.aspx. Zugegriffen: 15.2.2017.

11. Statistik Austria. Lebenserwartung. 2017. http://www. statistikaustria.at/web_de/statistiken/menschen_und _gesellschaft/bevoelkerung/sterbetafeln/index.html. Zugegriffen: 20.2.2017.

12. Kontis V, Bennett JE, Mathers CD, et al. Future life expectancy in 35 industrialized countries: projections with a Bayesian model ensemble. Lancet. 2017;389:1323-35. https://doi.org/10.1016/S0140-6736(16)32381-9.

13. United Nations Department of Economic and Social Affairs. Population Division. World population ageing 2015 (ST/ESA/SER.A/390). 2015. http://www. un.org/en/development/desa/population/publications/ pdf/ageing/WPA2015_Report.pdf.Zugegriffen:20.2.2017.

14. Poland GA, Jacobson RM, Ovsyannikova IG. Trends affecting the future of vaccine development and delivery: the role of demographics, regulatory science, the anti-vaccine movement, and vaccinomics. Vaccine. 2009;27:3240-4. https://doi.org/10.1016/j.vaccine.2009.01.069.

15. Rechel B, GrundyE, Robine LM, etal.Ageingin theEuropean Union. Lancet. 2013;381:1312-22.

16. Marengonia A, Anglemana S, Melisa R, et al. Aging with multimorbidity: asystematic review of theliterature.Ageing Res Rev. 2011;10:430-9. https://doi.org/10.1016/j.arr.2011. 03.003.

17. HeppnerHJ, SieberC, WalgerP, etal. Infectionsin the elderly. CritCare Clin. 2013;29:757-74.

18. Pearson-Stuttard J, Blundell S, Harris T, et al. Diabetes and infection: assessing the association with glycaemic control in population-based studies. Lancet Diabetes Endocrinol. 2016;4:148-58.

19. Doherty M, Schmidt-Ott R, Santos JI, et al. Vaccination of special populations: protecting the vulnerable. Vaccine. 2016;52:6681-90. https://doi.org/10.1016/j.vaccine. 2016.11.015.

20. Hutt HJ, Bennerscheidt P, Thiel B, et al. Immunseneszenz und Impfungen im höheren Lebensalter. Med Klin. 2010;105:802-7.

21. Kovaiou RD, Herndler-Brandstetter D, Grubeck-Loebenstein B. Age-related changes in immunity: implications for vaccination in the elderly. Expert Rev Mol Med.2007;9:1-17.

22. Lambert PH, Laurent PE. Intradermal vaccine delivery: will new delivery systems transform vaccine administration? Vaccine. 2008;26:3197-208.

23. Grimprel E, von Sonnenburg F, Sanger R, et al. Combined reduced-antigen-content diphtheria-tetanus-acellular pertussis and polio vaccine (dTpa-IPV) for booster vaccination of adults. Vaccine. 2005;23:3657-67.
24. van Damme P, Burgess M. Immunogenicity of a combined diphtheria-tetanus-acellular pertussis vaccine in adults. Vaccine. 2004;22:305-8.

25. Hoel T, Wolter JM, Schuerman LM. Combined diphtheriatetanus-pertussis vaccine for tetanus-prone wound management in adults. EurJ Emerg Med. 2006;13:67-71.

26. Vellinga A, Van DP, Joossens E, et al. Response to diphtheria booster vaccination in healthy adults: vaccine trial. BMJ. 2000;320:217.

27. Van DammeP, McIntyreP, GrimprelE, etal.Immunogenicity of the reduced-antigen-content dTpa vaccine (Boostrix $(\mathrm{R})$ ) in adults 55 years of age and over: a sub-analysis of four trials. Vaccine. 2011;29:5932-9.

28. Weinberger B, Schirmer M, Matteucci GR, et al. Recall responses to tetanus and diphtheria vaccination are frequently insufficient in elderly persons. PLOSONE. 2013;8:e82967.

29. Simon AK, Hollander GA, McMichael A. Evolution of the immune system in humans from infancy to old age. Proc Biol Sci. 2015;282:2014385. https://doi.org/10.1098/rspb. 2014.3085.

30. Michel JP, Lang O. Promoting life course vaccination. Rejuvenation Res. 2011;14:75-81. https://doi.org/10.1089/rej. 2010.1078 .

31. Pham H, GeraciSA, Burton MJ.CDCAdvisoryCommittee on Immunization Practices Adult immunizations: update on recommendations. Am J Med. 2011;124:698-701. https:// doi.org/10.1016/j.amjmed.2010.07.032.

32. Statistik Austria. Gebärmutterhalskrebs. 2017. http://www. statistik.at/web_de/statistiken/menschen_und_ gesellschaft/gesundheit/krebserkrankungen/gebaer mutterhals/index.html.Zugegriffen:23.3.2017.

33. Kristensen M, van Lier A, Eilers R, et al. Burden of four vaccine preventable diseases in older adults. Vaccine. 2016;34:942-9.

34. Yoshikawa TT. Epidemiology and unique aspects of aging and infectious diseases. Clin Infect Dis. 2000;30:931-3.

35. Gavazzi G, Krause KH. Ageing and infection. Lancet Infect Dis. 2002;11:659-66.

36. Jansen AG, Rodenburg GD, deGreeffSC, etal. Invasivepneumococcal disease in the Netherlands: syndromes, outcome and potential vaccine benefits. Vaccine. 2009;27:2394-401.

37. High KP, Bradley S, Loeb M, et al. A new paradigm for clinical investigation of infectious syndromes in older adults: assessment of functional status as a risk factor and outcome measure. Clin Infect Dis. 2005;40:114-22.

38. Johnson RW, Alvarez-Pasquin M, Bijl M, et al. Herpes zoster epidemiology, management, and disease and economic burden in Europe: a multidisciplinary perspective. Ther Adv Vaccines. 2015;4:109-20. https://doi.org/10. $1177 / 2051013615599151$.

39. Drolet M, Oxman MN, Levin MJ, et al. Vaccination against herpes zoster in developed countries. State of the evidence. Hum Vaccin Immunother. 2013;9:1177-84.

40. Oxman MN, Levin MJ, Johnson GR, et al. Shingles Prevention Study Group. A vaccine to prevent herpes zoster and postherpetic neuralgia in older adults. N Engl J Med. 2005;352:2271-84.

41. Schmader KE, Levin MJ, Gnann JW, etal.Efficacy, safety, and tolerability of herpes zoster vaccine in persons aged 50-59 years. Clin Infect Dis. 2012;54:922-8.

42. World Health Organization (WHO). Fact sheet Influenza No. 211. 2009. http://www.who.int/mediacentre/ factsheets $/ 2003 /$ fs $211 /$ en.

43. European Centre for Disease Prevention and Control (ECDC). Fact sheet for health professionals on seasonal influenza. 2017. https://ecdc.europa.eu/en/seasonalinfluenza/facts/key-messages. Zugegriffen:23.3.2017. 
44. Kuo HW, Schmid D, Liu YL, et al. Influenza-related excess mortality, Austria 2001 till 2009. Wien Klin Wochenschr. 2011;123:593-8. https://doi.org/10.1007/s00508011-0019-7.

45. Redlberger-Fritz M, Aberle JH, Popow-Kraupp T, et al. Attributable deaths due to influenza: a comparative study of seasonal and pandemic influenza. Eur J Epidemiol. 2012;27:567-75. https://doi.org/10.1007/s10654-0129701-y.

46. Kunze U, Böhm G, Groman E. Influenza vaccination in Austria, 1982-2011. Vaccine. 2013;44:5099-103. https:// doi.org/10.1016/j.vaccine.2013.08.050.

47. Bundesministerium für Gesundheit und Frauen. Impfplan Österreich 2017. 2017. http:/ / www.bmgf.gv.at/home/ Impfplan.Zugegriffen: 14.2.2017.

48. World Health Organization (WHO). Vaccines against Influenza-WHOposition paper.WklyEpidemiolRec. 2012;47:23.

49. World Health Organization (WHO). Factsheet measles. 2015. http://www.euro.who.int/en/media-centre/ sections/fact-sheets/2015/measles-in-the-whoeuropean-region. Zugegriffen:29.3.2017.

50. European Centre for Disease Prevention and Control (ECDC). Monthly measles epidemiological updates. 2017. http://ecdc.europa.eu/en/healthtopics/measles/ epidemiological_data/Pages/measles_surveillance_ reports.aspx.

51. Bundesministerium für Gesundheit und Frauen. Masernaktuelle Situation in Österreich. 2017. https:/ / www.bmgf. gv.at/home/Masern.Zugegriffen: 8.6.2017.

52. Muscat M. Who gets measles in Europe? J Infect Dis. 2011;(Suppl 1):S353-S65. https://doi.org/10.1093/infdis/ jir067.

53. World Health Organization (WHO). Immunization, vaccines and biologicals - pertussis. 2008. http://www.who.int/ immunization/monitoring_surveillance/burden/vpd/ surveillance_type/passive/pertussis/en/. Zugegriffen: 23.3.2017.

54. Zentrum für Reisemedizin. Pertussis. 2017. http://www. reisemed.at/krankheiten/keuchhusten-pertussis. Zugegriffen:23.3.2017.

55. Pilishvili T, Bennett NM. Pneumococcal disease prevention among adults: Strategies for the use of pneumococcal vaccines. Vaccine. 2015;33:D60-D5.

56. European Centre for Disease Prevention and Control (ECDC). Annual Epidemiological Report 2016 - Invasive pneumococcal disease. 2016. http://ecdc.europa.eu/en/ healthtopics/pneumococcal_infection/Pages/Annualepidemiological-report-2016.aspx. Zugegriffen:29.3.2017.

57. Österreichische Agentur für Gesundheit und Ernährungssicherheit (AGES), Institut für medizinische Mikrobiologie und HygieneGraz. JahresberichtNationale Referenzzentrale für Pneumokokken. 2014. https:/ / www.bmgf.gv.at/cms / home/attachments/8/7/9/CH1470/CMS1426082563865/ jb_pneumokokken_2014_-_format_elbel_10032015.pdf. Zugegriffen: 27.6.2017.

58. World Health Organization (WHO). WHO position paper - 23-valent pneumococcal polysaccharide vaccine. Wkly Epidemiol Rec. 2008;42:373-84.

59. Ewald H, Briel M, Vuichard D, et al. The clinical effectiveness of pneumococcal conjugate vaccines: a systematic review and meta-analysis of randomized controlled trials. Dtsch Arztebl Int. 2016;4113:139-46. https://doi.org/10. 3238/arztebl.2016.0139.

60. European Centre for Disease Prevention and Control (ECDC). Annual Epidemiological Report 2016 - Poliomyelitis. 2016. https://ecdc.europa.eu/en/publications- data/polio-annual-epidemiological-report-2016-2014data.Zugegriffen:9.3.2017.

61. Celentano LP, Lopalco PL, Huitric E, et al. Polio and the risk for the European Union. Lancet. 2014;383:216-7. https:// doi.org/10.1016/S0140-6736(13)62223-0.

62 . Weinberger B. Adult vaccination against tetanus and diphtheria: the European perspective. Clin Exp Immunol. 2017;187:93-9. https://doi.org/10.1111/cei.12822.

63. Di Giovine P, Kafatos G, Nardone A, et al. Comparative seroepidemiology of diphtheria in six European countries and Israel. Epidemiol Infect. 2013;141:132-42.

64. Chlibek R, Anca I, André F, et al. Adult vaccination in 11 Central European countries - Calendars are not just for children. Vaccine. 2012;30:1529-40.

65. VENICE (Vaccine European New Integrated Collaboration Effort) National Gatekeepers, Contact Points, Kanitz EE, Wu LA, Giambia C, et al. Variation in adult vaccination policies across Europe: an overview from VENICE network on vaccine recommendations, funding and coverage. Vaccine. 2012;30:5222-8.

66. European Federation of Internal Medicine ADVICE Working Group, Ozisik L, Tanriover TM, Rigby S. ADVICE for a healthier life: adult vaccination campaign in Europe. Eur J Intern Med.2016;33:14-20.

67. Wu LA, Kanitz E, Crumly J, et al. Adult immunization policies in advanced economies: vaccination recommendations, financing, and vaccination coverage. Int J Public Health. 2013;58:865-74. https://doi.org/10.1007/s00038012-0438-x.

68. Vaccine European New Integrated Collaboration Effort (Venice I-III). 2017. http://venice.cineca.org/the_project. html.Zugegriffen:9.3.2017.

69. Lopalco PL, Carrillo Sanstisteve P. Actual immunization coverage throughout Europe: are existing data sufficient? Clin Microbiol Infect. 2014;20:7-11.

70. PalacheA, Oriol-MathieuV, Fino M, etal. Seasonal influenza vaccine dose distribution in 195 countries (2004-2013): little progress in estimated global vaccination coverage. Vaccine. 2015;33:5598-605.

71. European Centre for Disease Prevention and Control (ECDC). Seasonal influenza vaccination and antiviral use in Europe - Overview of vaccination recommendations and coverage rates in the EU Member States for the 2013-14 and 2014-15 influenza season. 2016. http://ecdc.europa.eu/en/publications/_layouts/forms/ Publication_DispForm.aspx?List=4f55ad51-4aed-4d32b960-af70113dbb90\&ID=1528. Zugegriffen: 9.3.2017.

72. Hoffman K, Paget J, Wojczewski S, et al. Influenza vaccination prevalence and demographic factors of patients and GPs in primary care in Austria and Croatia: a cross-sectional comparative study in the framework of the APRES project. Eur J Public Health. 2016;26:395-401. https://doi.org/10. 1093/eurpub/ckw006.

73. Michel JP, Chidiac C, Grubeck-Loebenstein B, et al. Advocating vaccination of adults aged 60 years and older in Western Europe: statement by the Joint Vaccine Working Group of the European Union Geriatric Medicine Society and the International Association of Gerontology and Geriatrics-European Region. Rejuvenation Res. 2009;12:127-35. https://doi.org/10.1089/rej.2008.0813.

74. Esposito S, Bonanni P, Maggi S, et al. Recommended immunization schedules for adults: clinical practice guidelines by the Escmid Vaccine Study Group (EVASG), European Geriatric Medicine Society (EUGMS) and the World Association for Infectious Diseases and Immunological Disorders (WAidid). Hum Vaccin Immunother. 2016;12:1777-94. https:// doi.org/10.1080/21645515.2016.1150396. 
75. ESCMID Vaccine Study Group, Esposito S, Durando P, Bosis S, et al. ESCMID Vaccine Study Group. Vaccine-preventable diseases: from paediatric to adult targets. Eur J Intern Med. 2014;25:203-12.https://doi.org/10.1016/j.ejim.2013. 12.004 .

76. Eilers R, Krabbe PF, de Melker HE. Factors affecting the uptake of vaccination by the elderly in Western society. Prev Med. 2014;69:224-34. https://doi.org/10.1016/j. ypmed.2014.10.017.

77. Gnanasekaran G, Biedenbender R, Davidson HE, et al. Vaccinations for the older adult. Clin Geriatr Med. 2016;32:609-25.

78. Monto AS, Davenport FM, Napier JA, et al. Modification of an outbreak of influenza in Tecumseh, Michigan by vaccination of schoolchildren. J Infect Dis. 1970;122:16-25.

79. Reichert TA, Sugaya N, Fedson DS, et al. The Japanese experience with vaccinating schoolchildren against influenza. NEngl J Med. 2001;344:889-886.

80. Esposito S, Marchisio P, Cavagna R, et al. Effectiveness of influenza vaccination of children with recurrent respiratory tract infections in reducing respiratory-related morbidity within the households. Vaccine. 2003;21:3162-8.

81. Principi N, Esposito S, Marchisio P, et al. Socioeconomic impact of influenza on healthy children and their families. Pediatr Infect Dis J. 2003;22:S207-S10.

82. Loeb M, Russell ML, Moss L, et al. Effect of influenza vaccination of children on infection rates in Hutterite communities: a randomized trial.JAMA. 2010;303:943-50.

83. Tae Hyong K. Seasonal influenza and vaccine herd effect. Clin Exp Vaccine Res. 2014;3:128-32. https://doi.org/10. 7774/cevr.2014.3.2.128.

84. Moore MR, Link-Gelles R, Schaffner W, et al. Impact of 13Valent pneumococcal conjugate vaccine used in children on invasive pneumococcal disease in children and adults in the United States: analysis of Multisite, population-based surveillance. Lancet Infect Dis. 2015;15:301-9. https:// doi. org/10.1016/S1473-3099(14)71081-3.

85. Waight PA, Andrews NJ, Shamez N, et al. Effect of the 13 -valent pneumococcal conjugate vaccine on invasive pneumococcal disease in England and Wales 4 years after its introduction: an observational cohort study. Lancet Infect Dis. 2015;15:535-43.

86. Lal H, Cunningham AL, Godeaux O. Efficacy of an adjuvanted herpes zoster subunit vaccine in older adults. $\mathrm{N} \mathrm{Engl} \mathrm{J}$ Med. 2015;372:2087-96.

87. Tan L. Adult vaccination: now is the time to realize an unfulfilled potential.Hum Vaccin Immunother. 2015;11:2158-66. https://doi.org/10.4161/21645515.2014.982998.

88. Bundesministerium für Gesundheit und Frauen. Impfungen für Erwachsene im erwerbsfähigen Alter. Empfehlungen als Erweiterung des Österreichischen Impfplans. 2015. http://www.bmgf.gv.at/home/Gesundheit/ Gesundheitsfoerderung_Praevention/Impfen/ Impfungen_fuer_Erwachsene_im_erwerbsfaehigen_ Alter.Zugegriffen:30.3.2017.

89. Kurier. Masern-Häufung - was von einer Impfpflicht $\mathrm{zu}$ halten ist. 2017. https://kurier.at/wissen/masernhaeufung-was-von-einer-impfpflicht-zu-halten-ist/245. 193.108 (Erstellt: 7.Febr.2017).Zugegriffen: 7.2.2017.

90. Bundesministerium für Gesundheit und Frauen. Impfungen für MitarbeiterInnen des Gesundheitswesens. Empfehlungen als Erweiterung desÖsterreichischen Impfplans. 2012. http://www.bmgf.gv.at/home/Impfempfehlungen Gesundheitspersonal.Zugegriffen:30.3.2017. 